\title{
The common property of isotopic anomalies in meteorites
}

\author{
F. Robert ${ }^{\star}$ \\ Muséum-USM 205, CNRS-UMS 2679 LEME, 61 rue Buffon, 75005 Paris, France \\ Received 20 June 2003 / Accepted 6 November 2003

\begin{abstract}
The treatment proposed to account for the non-mass-dependent isotopic fractionation effect observed for oxygen isotopes during the synthesis of ozone (Robert \& Camy-Peyret 2001) is applied to other chemical elements. A numerical treatment to calculate isotopic reaction rate ratios is proposed. This treatment yields non-mass-dependent isotopic effects in other chemical elements, qualitatively similar to those observed in some of the high temperature minerals found in the carbonaceous meteorites. This treatment may reflect the numerical consequences of an unrecognized quantum mechanical effect, linked to a property of chemical reactions involving indistinguishable isotopes.
\end{abstract}

Key words. meteoroids - supernovae: general - nucleosynthesis - solar system: formation

\section{Introduction}

Non-mass-dependent isotopic effects (designated in the Cosmochemical literature as "isotopic anomalies") have been found in almost all the chemical elements (including the noble gases) of the refractory minerals of the carbonaceous meteorites. Because there is no experimental evidence that such effects can be achieved through chemical reactions, a nuclear origin for these isotopic anomalies has been postulated. This generally accepted interpretation of isotopic anomalies implies that interstellar minerals - often referred to as "pre-solar" -, preserved in meteorites, were condensed in envelopes of supernovae that predated the formation of the solar system (Clayton et al. 1973; Wasserburg \& Papanastassiou 1980; Ott 1993; Zinner 1995). Indeed, numerous astrophysical calculations have shown that these isotopic anomalies may be reconciled with the theoretical predictions of nucleosynthesis in stars. In some examples, remarkable agreements between meteorite data and nuclear physics calculations have been quantitatively achieved (see the extensive work of D. Clayton and his co-workers on this matter; for example Clayton et al. 1997, 2001).

In this paper, it will be shown that several anomalies share a common property which can be accounted for by a single formula describing the reaction rates of the different isotopomers of the same chemical element. Such a formula is based on a unique assumption: the rates involving reactions between indistinguishable isotopes are different from those involving distinguishable isotopes. This conclusion was reached (Robert et al. 1988; Robert \& Baudon 1990; Robert \& Camy-Peyret 2001) by studying the anomalous isotopic effect found during the synthesis of ozone (Mauersberger 1981; Mauersberger et al. 1999; Thiemens et al. 1999; Janssen et al. 2003;

\footnotetext{
^ e-mail: robert@mnhn.fr
}

Mauersberger et al. 2003) and ascribed to a non-massdependent isotopic effect (Thiemens \& Heidenreich III 1983).

Another theory accounting for the ozone effect has been proposed by Hathorn \& Marcus (1999, 2000; see also Gao \& Marcus 2001, 2002). In this theory, the non-mass-dependent isotopic fractionation effect is introduced as a free parameter which is supposed to be due to the fact that the density of quantum states of a vibrationally energetic molecule is greater for the symmetric than for the asymmetric molecules because there are fewer dynamical coupling terms in the symmetric than in the asymmetric molecules. Both theories reproduce quantitatively the individual reaction rates obtained by Mauersberger et al. (1999) and Janssen et al. (1999). However, the treatment by Robert \& Camy-Peyret (2001) predicts positive and negative isotopic anomalies according to the energy at which the energetic molecule is stabilized while the Marcus et al. theory seems to preclude such negative effects. Therefore, beside future laboratory experiments, the meteorite data may help to distinguish which of these two proposals is correct.

\section{Equations for isotopic rate constant ratio}

\subsection{Mass-dependent isotopic fractionation}

In the present approach, the individual reaction rates for the different isotopomers are not calculated and consequently the isotopic fractionation factor is a free parameter. The proposed formalism allows the calculation of all the possible relations between the reactions rates normalized to a reference reaction. That is to say: if the isotopic fractionation factor of one isotopomer relative to an other isotopomer used as a reference is known, all the other isotopic fractionation factors involving all the other isotopomers are known. An example is given here after. 
Let us consider a chemical element "I" having 3 stable isotopes, $\mathrm{X}, \mathrm{Y}$ and $\mathrm{Z}$ and 3 reactions among the 9 possible reactions involved in the reactions I+IM:

$$
\begin{array}{lll}
\mathrm{X}+\mathrm{XM} \rightarrow \mathrm{XXM} & & k_{\mathrm{X}-\mathrm{XM}} \\
X+\mathrm{YM} \rightarrow \mathrm{XYM} \quad(\text { or YXM) } & k_{\mathrm{X}-\mathrm{YM}} \\
\boldsymbol{Y}+\mathrm{XM} \rightarrow \mathrm{YXM} \quad \text { (or XYM) } & k_{\mathrm{Y}-\mathrm{XM}}
\end{array}
$$

$\mathrm{X}$ and $\mathrm{Y}$ are atoms, $\mathrm{XM}$ and $\mathrm{YM}$ are molecules, and $k$ are the overall rate constants which can be calculated by the usual quantum mechanical methods. Reactions take place between the incident isotope and the isotope in the M molecule. During the reaction, an isotope exchange may (or may not) takes place; hence the notation "or" in (2) and (3). Bold italic is used to designate the incident atom except for reaction (1) where it is not possible to decide which of the two isotopes $\mathrm{X}$ in the XXM molecule is the incident atom. The rates of XYM and YXM are counted separately. The rates can be written:

$$
\begin{aligned}
\mathrm{d}[\mathrm{XXM}] / \mathrm{d} t & =[\mathrm{X}][\mathrm{XM}] k_{\mathrm{X}-\mathrm{XM}} \\
\mathrm{d}[\mathrm{XYM}] / \mathrm{d} t & =[\mathrm{X}][\mathrm{YM}] k_{\mathrm{X}-\mathrm{YM}}+[\mathrm{Y}][\mathrm{XM}] k_{\mathrm{Y}-\mathrm{XM}}
\end{aligned}
$$

with number densities in brackets. To avoid the complex calculation for each $k$ value, the mass-dependent fractionation rule is written empirically:

$$
k_{\mathrm{X}-\mathrm{YM}} / k_{\mathrm{X}-\mathrm{XM}}=\left[\mu_{\mathrm{X}-\mathrm{YM}} / \mu_{\mathrm{X}-\mathrm{XM}}\right]^{a}
$$

with $\mu$ the reduced mass of the reactants. In such an approach, if the parameter " $a$ " is known, all the relations between all the rate constants are known. In the reaction rate ratio of (4) to (5) the number density of atoms and molecules vanishes and can be replaced by the relative abundance of the isotopes $\mathrm{X}$, and $\mathrm{Y}$, i.e. by $x$, and $y$, respectively with $x+y+z=1$ (for this 3 isotope system). Defining the isotopic fractionation factor $R$ as:

$$
R=\mathrm{d}[\mathrm{XYM}] / \mathrm{d}[\mathrm{XXM}]
$$

the numerator and denominator can be written as:

$$
\begin{aligned}
\mathrm{d}[\mathrm{XYM}] & +\mathrm{d}[\mathrm{YXM}] \\
& =\left\{x y\left[\mu_{\mathrm{X}-\mathrm{YM}} / \mu_{\mathrm{X}-\mathrm{XM}}\right]^{a}+\left\{y x\left[\mu_{\mathrm{Y}-\mathrm{XM}} / \mu_{\mathrm{X}-\mathrm{XM}}\right]^{a}\right\}\right. \\
\mathrm{d}[\mathrm{XXM}] & =\{x x\} .
\end{aligned}
$$

Since $x$ and $y$ are known (they are the relative natural abundance of the isotopes of a given chemical element), for a given value of " $a$ ", all the isotopic fractionation factors for all the possible substitutions of X, Y and Z can be calculated. Such a method reproduces with a high degree of precision the results obtained by quantum mechanical calculations.

A numerical example is given in Table 1 for the 3 isotopes of oxygen. In Table 1, all individual rates are calculated and the total abundance of each isotope in all possible isotopomers are calculated. Let us take the example of the calculation of the relative abundance of ${ }^{16} \mathrm{O}$ in the final molecules OOM with $x_{16}$, $x_{17}$ and $x_{18}$ the relative natural abundance of ${ }^{16} \mathrm{O},{ }^{17} \mathrm{O}$ and ${ }^{18} \mathrm{O}$ in the reactants and $x_{16}^{\prime}, x_{17}^{\prime}$ and $x_{18}^{\prime}$ the abundance of ${ }^{16} \mathrm{O},{ }^{17} \mathrm{O}$ and ${ }^{18} \mathrm{O}$ in OOM (O in OOM stands for ${ }^{16} \mathrm{O},{ }^{17} \mathrm{O}$ or $\left.{ }^{18} \mathrm{O}\right)$.

$x_{16}^{\prime}=\left[{ }^{16} \mathrm{O}^{6} \mathrm{OM}\right]+1 / 2\left[{ }^{16} \mathrm{O}^{17} \mathrm{OM}\right]+1 / 2\left[{ }^{16} \mathrm{O}^{18} \mathrm{OM}\right]$.

The factor $1 / 2$ introduced in (10) is the classical symmetry number standing for the fact that the molecular abundance is twice the elemental abundance of ${ }^{16} \mathrm{O}$ in the ${ }^{16} \mathrm{O}^{17} \mathrm{OM}$ and ${ }^{16} \mathrm{O}^{18} \mathrm{OM}$ molecules.

In isotopic ratios, the term in (10) appears as:

$$
\begin{array}{r}
x_{16}^{\prime}=x_{16} x_{16}+1 / 2\left\{x_{16} x_{17} \Delta \mu_{17-16 \mathrm{M}}^{a}+x_{16} x_{18} \Delta \mu_{18-16 \mathrm{M}}^{a}\right. \\
\left.+x_{16} x_{17} \Delta \mu_{16-17 \mathrm{M}}^{a}+x_{16} x_{18} \Delta \mu_{16-18 \mathrm{M}}^{a}\right\}
\end{array}
$$

with $\Delta \mu_{\mathrm{X}-\mathrm{YM}}^{a}=\left[\mu_{\mathrm{X}-\mathrm{YM}} / \mu_{16-16 \mathrm{M}}\right]^{a}$

and similarly for $x_{17}^{\prime}$ and $x_{18}^{\prime}$. If no isotopic fractionation takes place (i.e. for $a=0$ ) the sum $x_{16}^{\prime}+x_{17}^{\prime}+x_{18}^{\prime}=1$ indicating that the isotopic composition of the product OOM is exactly equal to the isotopic compositions of the reactants. If $a \neq 0$, the ${ }^{17} \mathrm{O} /{ }^{16} \mathrm{O}$ and ${ }^{18} \mathrm{O} /{ }^{16} \mathrm{O}$ ratios of the OOM molecule can be calculated and expressed in their corresponding usual delta notations i.e.:

$\delta^{17} \mathrm{O}(\%)=\left\{\left[x_{17}^{\prime} / x_{16}^{\prime}\right] /\left[x_{17} / x_{16}\right]-1\right\} \times 1000$

and similarly for $\delta^{18} \mathrm{O}(\%)$.

In Table 1 , the calculated ratio $\delta^{17} \mathrm{O} / \delta^{18} \mathrm{O}$ is 0.513 , in close agreement with the experimental or theoretical ratio. Note that the $\delta^{17} \mathrm{O} / \delta^{18} \mathrm{O}$ ratio is independent of the value $x_{16}, x_{17}$ and $x_{18}$ and almost independent of the value of the mass $\mathrm{M}$ constituting the molecule OOM. Therefore this method can be applied to all the chemical elements and will be used all along this paper as a "proxy" for the mass-dependent relations between rate constants which only differ by the mass of their isotopically substituted molecules.

In Sect. 2.2 discussing the non-mass-dependent isotopic fractionation, the $\delta^{17} \mathrm{O} / \delta^{18} \mathrm{O}$ ratio is no longer independent of the value $x_{16}, x_{17}$ and $x_{18}$; therefore the notation of the relative isotopic abundances were explicitly maintained in (11).

\subsection{Non-mass-dependent isotopic fractionation}

The aim of this section is to propose a treatment that permits the calculation of a non-mass-dependent isotopic fractionation effect in a manner general to that described in Sect. 2.1. Similar to the treatment of the mass-dependent fractionation proposed in the previous Sect. 2.1, no specific chemical reaction is assigned for such a calculation.

This treatment is based on a single assumption: the rate constant for the formation of molecules involving reactions between two indistinguishable isotopes (such as $\mathrm{X}+\mathrm{XM}$ ) are not related in a mass-dependent manner to the rate constants involving reactions between two distinguishable isotopes (such as $\mathrm{Y}+\mathrm{XM})$. Such a difference does not appear in reactions such as (1) to (3) but in 3-body reaction scheme where the formation of IIM occurs when the stabilization of the energetic molecule 
Table 1. A numerical example showing the mass-dependent relations between the different reaction rates in the oxygen isotopic system. In successive columns: (1) the isotopic reaction ${ }^{i} \mathrm{O}+{ }^{j} \mathrm{OM} \rightarrow{ }^{i} \mathrm{O}^{j} \mathrm{OM}$; (2) the reduced mass of the reactants for $M=5$; (3) the reduced mass ratio $\Delta \mu_{\mathrm{Y}-\mathrm{MX}}^{a}$ normalized to the reaction ${ }^{16} \mathrm{O}+{ }^{16} \mathrm{OM}$ for $a=1$; (4) the product of the relative abundance with ${ }^{16} \mathrm{O}=0.9976,{ }^{17} \mathrm{O}=4 \times 10^{-4}$ and ${ }^{18} \mathrm{O}=2 \times 10^{-3}$; (5) the product of $(3) \times(4)$. The ${ }^{17} \mathrm{O} /{ }^{16} \mathrm{O}$ ratio $\left(x_{17}^{\prime} / x_{16}^{\prime}\right)$ of the OOM molecule (noted $\left[{ }^{17} \mathrm{O} /{ }^{16} \mathrm{O}\right]_{\mathrm{oom}}$ ) is calculated from the values of the column (5); that is: $x_{16}^{\prime}=\left\{1 / 2\left[4.128 \times 10^{-4}+2.129 \times 10^{-3}+4.070 \times 10^{-4}+2.073 \times 10^{-3}\right]+9.952 \times 10^{-1}\right\}$. Similarly for $x_{17}^{\prime}$ and $x_{18}^{\prime}$. This gives: $\left[{ }^{17} \mathrm{O} /{ }^{16} \mathrm{O}\right]_{\mathrm{OOM}}=\left(x_{17}^{\prime} / x_{16}^{\prime}\right)=4.119 \times 10^{-4}$ and $\left[{ }^{18} \mathrm{O} /{ }^{16} \mathrm{O}\right]_{\mathrm{OOM}}=\left(x_{18}^{\prime} / x_{16}^{\prime}\right)=2.111 \times 10^{-3}$ corresponding to $\delta^{17} \mathrm{O}=$ $\left(\left[4.119 \times 10^{-4} /\left(4 \times 10^{-4} / 0.9976\right)\right]-1\right) \times 1000=+27.3 \%$ and similarly $\delta^{18} \mathrm{O}=+53.2 \%$. The slope in the diagram $\delta^{17} \mathrm{O}$ versus $\delta^{18} \mathrm{O}$ is equal to 0.513 .

\begin{tabular}{cclll}
\hline \hline$[1]$ & {$[2]$} & \multicolumn{1}{c}{$[3]$} & \multicolumn{1}{c}{$[4]$} & {$[5]$} \\
\hline${ }^{16} \mathrm{O}+{ }^{16} \mathrm{OM}$ & 9.081 & $\equiv 1$ & 0.9952 & 0.9952 \\
${ }^{17} \mathrm{O}+{ }^{16} \mathrm{OM}$ & 9.395 & 1.0345 & $3.990 \times 10^{-4}$ & $4.128 \times 10^{-4}$ \\
${ }^{18} \mathrm{O}+{ }^{16} \mathrm{OM}$ & 9.692 & 1.0673 & $1.995 \times 10^{-3}$ & $2.129 \times 10^{-3}$ \\
${ }^{16} \mathrm{O}+{ }^{17} \mathrm{OM}$ & 9.263 & 1.0201 & $3.990 \times 10^{-4}$ & $4.070 \times 10^{-4}$ \\
${ }^{17} \mathrm{O}+{ }^{17} \mathrm{OM}$ & 9.590 & 1.0560 & $1.600 \times 10^{-7}$ & $1.690 \times 10^{-7}$ \\
${ }^{18} \mathrm{O}+{ }^{17} \mathrm{OM}$ & 9.900 & 1.0902 & $8.000 \times 10^{-7}$ & $8.721 \times 10^{-7}$ \\
${ }^{16} \mathrm{O}+{ }^{18} \mathrm{OM}$ & 9.436 & 1.0391 & $1.995 \times 10^{-3}$ & $2.073 \times 10^{-3}$ \\
${ }^{17} \mathrm{O}+{ }^{18} \mathrm{OM}$ & 9.775 & 1.0764 & $8.000 \times 10^{-7}$ & $8.611 \times 10^{-7}$ \\
${ }^{18} \mathrm{O}+{ }^{18} \mathrm{OM}$ & 10.10 & 1.1119 & $4.000 \times 10^{-6}$ & $4.448 \times 10^{-6}$ \\
\hline
\end{tabular}

IIM* involved two channels:

$\begin{array}{ll}\mathrm{I}+\mathrm{IM} \rightarrow \mathrm{IIM}^{*} & k_{\mathrm{I}-\mathrm{IM}}^{*} \\ \mathrm{IIM}^{*} \rightarrow \mathrm{I}+\mathrm{IM} & k_{\mathrm{D}, \mathrm{I}-\mathrm{IM}} \\ \mathrm{IIM}^{*}+\mathrm{N} \rightarrow \mathrm{IIM}+\mathrm{N} & k_{\mathrm{N}} .\end{array}$

Equation (14) describes the formation of the energetic molecule IIM* with the rate constant $k^{*}$. Equation (15) represents the spontaneous dissociation of the energetic molecule with the rate constant $k_{\mathrm{D}}$ ( $\mathrm{D}$ for dissociation); its inverse $1 / k_{\mathrm{D}}$ characterizes the lifetime of the energetic molecule. Equation (16) corresponds to the possible stabilization of IIM* by a third body $\mathrm{N}$ bringing out the proper amount of internal energy. For simplicity it is assumed that no isotopic effect exists for $k_{\mathrm{N}}$ and thus no subscript is indicated in $k_{\mathrm{N}}$. The overall formation rate can be derived assuming that the concentration of the energetic molecule IIM* is constant (steady state). Two examples of solutions are given for two isotopic reactions involving $\mathrm{X}$ and $\mathrm{Y}$ :

$\mathrm{d}\left[\mathrm{XXM}^{*}\right] / \mathrm{d} t=0$.

Under this condition we have:

$\mathrm{d}[\mathrm{XXM}] / \mathrm{d} t=[\mathrm{X}][\mathrm{XM}]\left\{\mathrm{k}_{\mathrm{X}-\mathrm{XM}}^{*} k_{\mathrm{N}}[\mathrm{N}] /\left(k_{\mathrm{D}, \mathrm{X}-\mathrm{XM}}+k_{\mathrm{N}}[\mathrm{N}]\right)\right\}$

and similarly for YXM:

$\mathrm{d}[\mathrm{YXM}] / \mathrm{d} t=[\mathrm{Y}][\mathrm{XM}]\left\{k_{\mathrm{Y}-\mathrm{XM}}^{*} k_{\mathrm{N}}[\mathrm{N}] /\left(k_{\mathrm{D}, \mathrm{Y}-\mathrm{XM}}+k_{\mathrm{N}}[\mathrm{N}]\right)\right\}$.

According to our assumption $k_{\mathrm{D}, \mathrm{X}-\mathrm{XM}}$ in (18) and $k_{\mathrm{D}, \mathrm{Y}-\mathrm{XM}}$ in (19) are not related in a mass-dependent manner. As proposed to interpret the results on ozone, $k_{\mathrm{D}, \mathrm{X}-\mathrm{XM}}$ and $k_{\mathrm{D}, \mathrm{Y}-\mathrm{XM}}$ are written as:

$k_{\mathrm{D}, \mathrm{X}-\mathrm{XM}}=1 / 2\left[k_{1, \mathrm{X}-\mathrm{XM}}+k_{2, \mathrm{X}-\mathrm{XM}}\right]$

$k_{\mathrm{D}, \mathrm{Y}-\mathrm{XM}}=k_{1, \mathrm{Y}-\mathrm{XM}}$.
The additional term $k_{2}$ appearing to describe the spontaneous dissociation rate of an energetic molecule formed by reactions involving indistinguishable isotopes is at the origin of the nonmass-dependent effect. The factor $1 / 2$ is introduced in (20) to re-normalize the collision rate to the same number of reactions involving dis- and indistinguishable isotopes. An interpretation of this formalism is proposed in Robert \& Camy Peyret (2001; see also Appendix).

Let us consider the reactions yielding the formation of the 3 stable molecules XXM, YXM and YYM; all the other possible rates involving all the other isotopomers can be derived from these 3 reactions. The two isotopic ratios $R 1=[\mathrm{YXM}] /[\mathrm{XXM}]$ and $R 2=[\mathrm{YYM}] /[\mathrm{XXM}]$ with the reaction $\mathrm{X}+\mathrm{XM}$ used to normalize the isotopic rate ratios are now calculated. To ease the discussion of the numerical results, we will consider situations where the "low pressure approximation" (i.e. $k_{\mathrm{N}}[\mathrm{N}] \ll$ $\left.k_{\mathrm{D}}\right)$ applies; that is:

$R 1=\left\{[\mathrm{Y}][\mathrm{XM}] k_{\mathrm{Y}-\mathrm{XM}}^{*} k_{\mathrm{D}, \mathrm{X}-\mathrm{XM}}\right\} /\left\{[\mathrm{X}][\mathrm{XM}] k_{\mathrm{X}-\mathrm{XM}}^{*} k_{\mathrm{D}, \mathrm{Y}-\mathrm{XM}}\right\} .(22)$

In $R 1$, the ratio $k_{2}, k_{1}$ re-normalized to the same mass, is noted $\kappa$ :

$k_{2,} / k_{1}=\kappa$

As expected from the numerical results on ozone (Janssen et al. 2001) the parameter $\kappa$ is mass-dependent and is written as:

$k_{2, \mathrm{X}-\mathrm{XM}} / k_{1, \mathrm{Y}-\mathrm{XM}}=\kappa \Delta \mu_{\mathrm{Y}-\mathrm{XM}}^{c}$.

The rate constant ratio of $k^{*}$ and $k_{1}$ are related in a manner similar to that described in Sect. 2.1. That is:

$k_{\mathrm{Y}-\mathrm{XM}}^{*} / k_{\mathrm{X}-\mathrm{XM}}^{*}=\Delta \mu_{\mathrm{Y}-\mathrm{XM}}^{a}$

$k_{1, \mathrm{X}-\mathrm{XM}} / k_{1, \mathrm{Y}-\mathrm{XM}}=\Delta \mu_{\mathrm{Y}-\mathrm{XM}}^{b}$.

The mass-dependent fractionation between the constants are a priori different; hence the different parameters $a, b$ and $c$. 
The ratios $R 1$ is:

$$
\begin{aligned}
R 1 & =[\mathrm{YXM}] /[\mathrm{XXM}] \\
& =y x\left\{\Delta \mu_{\mathrm{Y}-\mathrm{XM}}^{a} 1 / 2\left[\Delta \mu_{\mathrm{Y}-\mathrm{XM}}^{b}+\kappa \Delta \mu_{\mathrm{Y}-\mathrm{XM}}^{c}\right]\right\} / x x .
\end{aligned}
$$

In $R 2$, the mass dependence between $k_{2}$ is similar to (26) and thus:

$R 2=[\mathrm{YYM}] /[\mathrm{XXM}]=y y\left\{\Delta \mu_{\mathrm{Y}-\mathrm{YM}}^{a} \Delta \mu_{\mathrm{Y}-\mathrm{YM}}^{b}\right\} / x x$.

The second isotopomer XYM resulting from the reaction $\mathrm{X}+\mathrm{YM}$ can be calculated similarly to (27). The abundance of the given isotope $\mathrm{X}$ in all the isotopomers IIM should be performed using the rules defined in (10) and (11). The classical mass-dependent isotopic effect is restored when one of the channels (15) or (16) is closed or when $k_{1}=k_{2}$ (giving $\kappa \approx 1$; see Apendix). When one of the two channels is closed the situation is similar to reactions (1) to (3). Considering a third isotope Z, Eq. (28) predicts a simple mass-dependent relation between the ratios involving the symmetrical isotopomers i.e. between $[\mathrm{YYM}] /[\mathrm{XXM}]$ and $[\mathrm{ZZM}] /[\mathrm{XXM}]$. This is observed in ozone.

Note that, contrary to the mass-dependent fractionation, the abundances of the isotopes $(x, y$ and $z$ ) do not vanish in the final isotopic ratios of the molecule IIM stabilized via reaction (16). In this respect, the non-mass-dependent fractionation can be defined as "abundance dependent", because the massdependent effects are second order contributions to the overall fractionation.

The parameter " $a$ " stands for the classical isotopic fractionation factor under equilibrium between the reactants and the energetic molecule. The parameter " $b$ " stands for the massdependent fractionation occurring during the dissociation of the energetic molecule. The parameter " $c$ " has no counterpart in the classical theory of isotopic fractionation. The study of ozone has shown that these parameters were constant through the various conditions of the ozone synthesis. In the minerals of meteorites, these conditions are unknown and these parameters will be considered as free.

When the low pressure approximation does not apply, the isotopic variations with pressure can be reproduced by such a formalism since $k_{\mathrm{N}}[\mathrm{N}] / k_{\mathrm{D}}$ is proportional to $P / P_{0}$ with $P$ the pressure at which the energetic molecule is stabilized (see the calculation of $P / P_{0}$ in Robert and Camy-Peyret). Defining

$$
k_{\mathrm{N}}[\mathrm{N}] / k_{\mathrm{D}} \propto P / P_{0}
$$

we have:

$$
\begin{aligned}
{[\mathrm{YXM}] /[\mathrm{XXM}] \approx\left\{y x \Delta \mu_{\mathrm{Y}-\mathrm{XM}}^{a}[\right.} & 1 / 2\left[\Delta \mu_{\mathrm{Y}-\mathrm{XM}}^{b}+\kappa \Delta \mu_{\mathrm{Y}-\mathrm{XM}}^{c}\right] \\
& \left.\left.+P / P_{0}\right]\right\} /\left\{x x\left[1+P / P_{0}\right]\right\} .
\end{aligned}
$$

The YYM/XXM ratio can be derived similarly and is close to (30). According to (30), when the pressure $P$ increases relative to $P_{0}$, the classical mass-dependent fractionation is progressively restored. Such a dependence with pressure of the anomalous isotopic fractionation is also observed in ozone. A simulation of this pressure effect will be given in Sect. 3 for silicon isotopes.

\section{Numerical application of the theory and comparison with meteorite data}

The aim of this section is to compare the theoretical predictions proposed in Sect. 2.2. with the results obtained for different chemical elements. The isotopic anomalies observed in meteorites are used for that purpose. However, it should be noted that in meteorites, isotopic anomalies are mostly found in high temperature minerals (condensed from a gas at temperature between 1500 and $2200 \mathrm{~K}$ ), suggesting that the conditions (temperature and pressure) under which these isotopic anomalies were established in meteorites are quite different from those prevailing during the ozone synthesis.

\subsection{Significance of the parameters}

The numerical results reported in this section were obtained for different values of the parameter $\kappa$ and different values of the parameters " $a$ ", " $b$ " and " $c$ " which dictate the mass dependence between the constants. According to the interpretation of (20), $\kappa$ can vary between 0 and $\gg 1$ (see Robert \& Camy-Peyret 2001). Thus, this type of non-mass-dependent isotopic fractionation can yield both positive (for $\kappa \geq 1$ ) and negative (for $\kappa \leq 1$ ) $\delta$ values.

The parameter " $a$ " dictates the isotopic fractionation between atoms and molecules. In ozone, it has been experimentally shown (Mauersberger 1999) that this fractionation is governed by the isotopic equilibrium between atoms and molecules, the number of collisions yielding an isotopic exchange between $\mathrm{O}$ and $\mathrm{O}_{2}$ greatly exceeding those forming $\mathrm{O}_{3}$ : $\mathrm{O}$ atoms have a $\delta^{18} \mathrm{O}=-70 \%$ and a $\delta^{17} \mathrm{O}=-36 \%$, relative to $\mathrm{O}_{2}$. In meteoritic minerals, such a pseudo isotopic equilibrium between atoms and molecules is not necessarily the case because the source of the atoms may be decoupled from that of the molecules (as for example when a source of atoms - stellar winds in astronomical situations (?) - interfere with a cold molecular medium). This remark is important for heavy elements (>30 amu) which are known to exhibit almost no massdependent isotopic fractionation at high temperature (i.e. $a \approx 0$ or $\delta \approx 0 \%$ ). Thus, in astronomical conditions, the parameter " $a$ " may a priori greatly exceed that expected from equilibrium or kinetic reactions between atoms and molecules.

\subsection{Implicit assumptions}

Two implicit assumptions are made in the numerical applications (these assumptions are classical in geochemistry). (1) Atoms and molecules are considered as infinite reservoirs. In other terms, the isotopic compositions of the reactants are constant in time. (2) The possible mixing in the meteoritic matter of several minerals carrying distinct isotopic anomalies is ignored. Nevertheless this complex situation does occur in the meteoritic matter, as illustrated by the grain size of the carriers of isotopic anomalies whose dimensions - in some examples do not exceed 100 nanometers. In such situations an isotopic composition cannot be assigned (and measured) for each individual grain. 
Table 2. A numerical example showing the non-mass-dependent relations between the different reaction rates in the oxygen isotopic system. In successive columns: (1) the isotopic reaction ${ }^{i} \mathrm{O}+{ }^{j} \mathrm{OM} \rightarrow{ }^{i} \mathrm{O}^{j} \mathrm{OM}$; (2) the reduced mass ratio $\Delta \mu_{\mathrm{Y}-\mathrm{MX}}^{a}=\left[\mu_{\mathrm{X}-\mathrm{MY}} / \mu_{\mathrm{X}-\mathrm{MX}}\right]^{a}$ for $a=0.1$ and $M=5$; (3) $\Delta \mu_{\mathrm{Y}-\mathrm{MX}}^{b}$ for $b=0.2$; (4) $\Delta \mu_{\mathrm{Y}-\mathrm{MX}}^{c}$ for $c=0.1$; (5) the reaction rate ratio $R$. Two situations are distinguished: (1) for ${ }^{i} \mathrm{O}+{ }^{i} \mathrm{OM}(i \equiv j)$, $R=\Delta \mu_{\mathrm{Y}-\mathrm{MY}}^{a} \Delta \mu_{\mathrm{Y}-\mathrm{MY}}^{b}$ and for $(2){ }^{j} \mathrm{O}+{ }^{i} \mathrm{OM}(i \neq j), R=\left\{\Delta \mu_{\mathrm{Y}-\mathrm{MX}}^{a} 1 / 2\left[\Delta \mu_{\mathrm{Y}-\mathrm{MX}}^{b}+\kappa \Delta \mu_{\mathrm{Y}-\mathrm{MX}}^{c}\right]\right.$; calculations are performed for $\kappa=0.8(6) \mathrm{The}$ product of the relative abundance with ${ }^{16} \mathrm{O}=0.9976,{ }^{17} \mathrm{O}=4 \times 10^{-4}$ and ${ }^{18} \mathrm{O}=2 \times 10^{-3}$ (7) the product of (5) $\times(6)$. The ${ }^{17} \mathrm{O} /{ }^{16} \mathrm{O}$ ratio of the OOM molecule (noted $\left[{ }^{17} \mathrm{O} /{ }^{16} \mathrm{O}\right]_{\mathrm{OOM}}$ ) is calculated from the values of the column [7]; that is for the abundance of ${ }^{16} \mathrm{O}:\left\{1 / 2\left[3.585 \times 10^{-4}+\right.\right.$ $\left.\left.1.789 \times 10^{-3}+3.587 \times 10^{-4}+1.792 \times 10^{-3}\right]+9.952 \times 10^{-1}\right\}$ and similarly for ${ }^{17} \mathrm{O}$ and ${ }^{18} \mathrm{O}$. The $\delta^{17} \mathrm{O}(\%)=\left(\left[{ }^{17} \mathrm{O} /{ }^{16} \mathrm{O}\right]_{\mathrm{OOM}} /[0.0004 / 0.9976]\right)-$ 1) $\times 1000$; similarly for $\delta^{18} \mathrm{O}\left(\%\right.$ ) . That is: $\delta^{17} \mathrm{O}=-101.1 \%$ and $\delta^{18} \mathrm{O}=-102.2 \%$ with $\delta^{17} \mathrm{O} / \delta^{18} \mathrm{O}=0.989$.

\begin{tabular}{cllllll}
\hline \hline$[1]$ & {$[2]$} & {$[3]$} & {$[4]$} & {$[5]$} & {$[6]$} & {$[7]$} \\
\hline${ }^{16} \mathrm{O}+{ }^{16} \mathrm{OM}$ & $\equiv 1$ & $\equiv 1$ & $\equiv 1$ & $\equiv 1$ & 0.9952 & 0.9952 \\
${ }^{16} \mathrm{O}+{ }^{17} \mathrm{OM}$ & 1.0034 & 0.9932 & 0.9966 & 0.8983 & $3.990 \times 10^{-4}$ & $3.585 \times 10^{-4}$ \\
${ }^{16} \mathrm{O}+{ }^{18} \mathrm{OM}$ & 1.0065 & 0.9871 & 0.9935 & 0.8968 & $1.995 \times 10^{-3}$ & $1.789 \times 10^{-3}$ \\
${ }^{17} \mathrm{O}+{ }^{16} \mathrm{OM}$ & 1.0020 & 0.9960 & 0.9980 & 0.8990 & $3.990 \times 10^{-4}$ & $3.587 \times 10^{-4}$ \\
${ }^{17} \mathrm{O}+{ }^{17} \mathrm{OM}$ & 1.0055 & 0.9892 & 0.9946 & 0.9946 & $1.600 \times 10^{-7}$ & $1.591 \times 10^{-7}$ \\
${ }^{17} \mathrm{O}+{ }^{18} \mathrm{OM}$ & 1.0087 & 0.9829 & 0.9914 & 0.8957 & $8.000 \times 10^{-7}$ & $7.166 \times 10^{-7}$ \\
${ }^{18} \mathrm{O}+{ }^{16} \mathrm{OM}$ & 1.0038 & 0.9924 & 0.9962 & 0.8981 & $1.995 \times 10^{-3}$ & $1.792 \times 10^{-3}$ \\
${ }^{18} \mathrm{O}+{ }^{17} \mathrm{OM}$ & 1.0074 & 0.9854 & 0.9927 & 0.8963 & $8.000 \times 10^{-7}$ & $7.171 \times 10^{-7}$ \\
${ }^{18} \mathrm{O}+{ }^{18} \mathrm{OM}$ & 1.0107 & 0.9790 & 0.9894 & 0.9894 & $4.000 \times 10^{-6}$ & $3.958 \times 10^{-6}$ \\
\hline
\end{tabular}

\subsection{Results}

In the following numerical calculations, the approximations made in Eqs. (27) and (28) will be used. For clarity, an example of a complete calculation is reported in Table 2. From this example it is possible to derived all the other numerical applications for the other isotopic systems reported in the Figs. 1 to 4 .

The results are reported in the 3 usual units used in Cosmochemistry: in $\delta$ units, in $\varepsilon$ units $(\varepsilon=\delta \times 10)$ and in $\varepsilon$ units using the normalization to two isotopes. In this last unit, because of analytical limitations, the absolute $\varepsilon$ values cannot be determined and another isotope is used as a second reference. In this unit, the isotopic compositions of the two reference isotopic ratios are assumed to be related in a massdependent manner and the isotopic compositions of the other masses are corrected by this mass-dependent term. In this unit, the mass-dependent fractionation yields $\varepsilon=0$ at all masses.

In these 3 units, the different diagrams reported in the Figs. 1 to 4 have the following properties.

1. In the $\delta$ versus $\delta$ diagrams, the mass-dependent fractionation should follow a linear relationship, the slope of which is dictated by the difference in mass of the isotopes (as for example, the slope $\approx 1 / 2$ in the oxygen diagram with $\delta^{17} \mathrm{O}$ in the $y$ axis and $\delta^{18} \mathrm{O}$ in the $x$ axis).

2. In $\delta$ or $\varepsilon$ units versus the mass of the isotopes, the massdependent fractionation should follow a linear relationship, the slope of which being the isotopic fractionation factor expressed per mass unit. In such diagrams, the slope can be positive or negative.

3. In the double normalization diagram versus the mass of the isotopes, the mass-dependent fractionation should give a flat pattern i.e. $\varepsilon=0 \%$ at all masses.

In the Cosmochemical literature, all data that depart from these mass-dependent relations are attributed to isotopic anomalies i.e. to nuclear effects.
Three different types of examples are distinguished in the Figs. 1 to 3. In Fig. 1, some of the classic examples of the Cosmochemical literature are reported and compared with the present numerical simulations. In Fig. 2, an example of the pressure effect is given for the silicon isotopes and compared with the meteorite data. In Fig. 3, two examples of meteorite data that cannot be reproduced by the present numerical simulation are shown. This may illustrate the limitations of our various assumptions (and especially the chosen mass-dependent relations) or the fact that these anomalies are purely nuclear or - of course - that the present theory is incorrect...

As far as the adjustments of the parameters of the calculation that fit the meteorite data are concerned two remarks should be made: (1) In most examples the parameter " $a$ " plays the same role as the parameter " $b$ "; hence " $a$ " can be generally assigned to be equal to 0 . Thus, the calculated patterns reported in the figures depend only on 3 free parameters: $\kappa, b$ and $c$ (2); Often - not always - the patterns can be translated to much higher $\delta$ units without changing the general shape of the patterns. This translation is obtained by multiplying the parameters by a constant factor. This indicates that the dilution of the measured anomalous isotopic composition by a "normal" (i.e. not fractionated or mass fractionated) composition does not change the predictions of the theory.

\section{Possible chemical reactions at the origin of the non-mass-dependent isotopic fractionation effect}

In this section we give a few examples of possible reactions yielding non-mass-dependent isotopic effects and which could be studied in the laboratory. These chemical systems are difficult to predict for three reasons: (1) the formula of the energetic molecules that can be formed during a given reaction are poorly known; (2) the determination of their lifetime is a difficult theoretical task and (3) this lifetime should be long enough in order for the energetic molecule to be involved in a second reaction, 

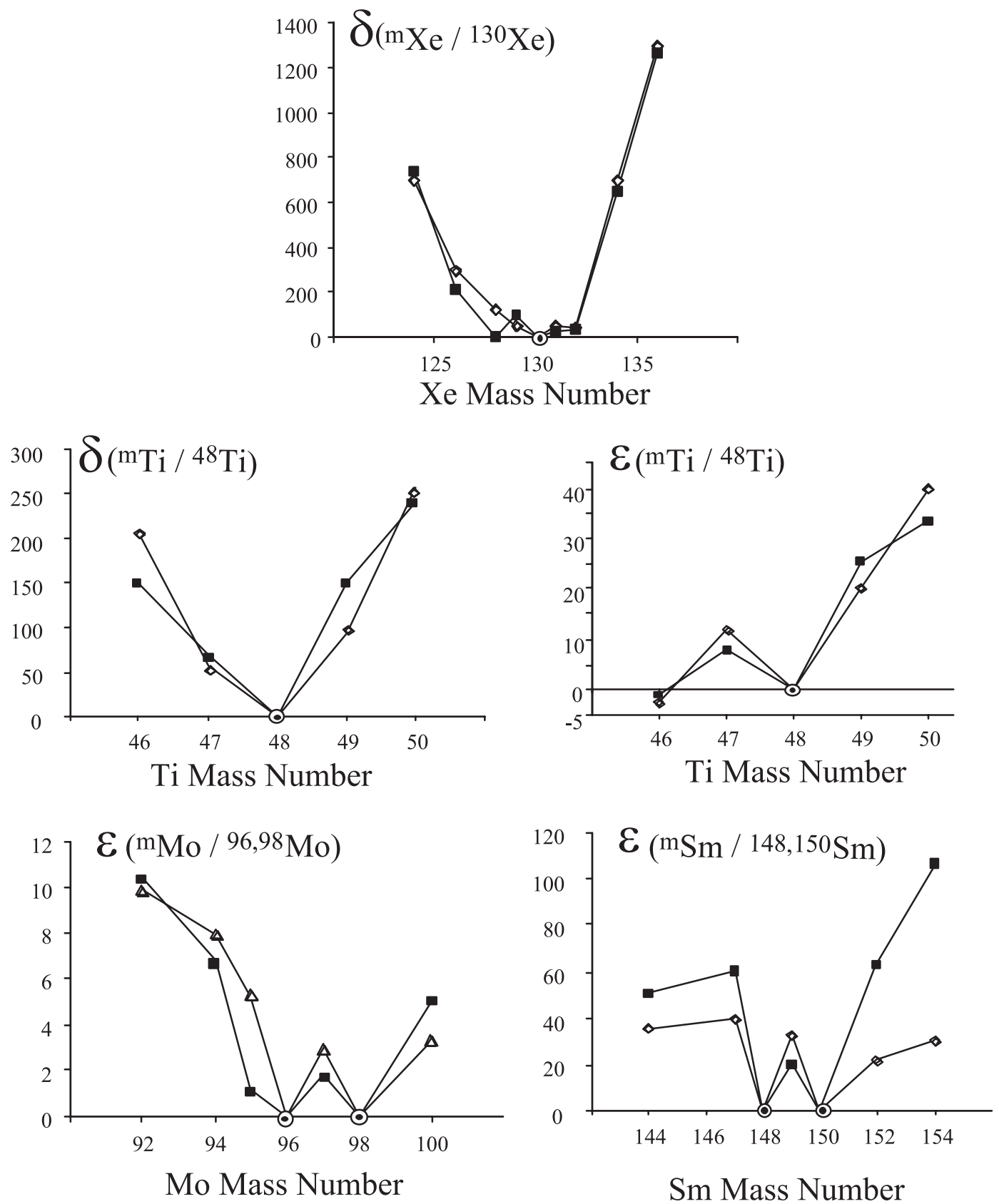

Fig. 1. Five examples of isotopic anomalies measured in meteorites are compared with the present calculations (black dots for calculations and open symbols for data). The calculated patterns were adjusted to the measured values through the parameters $\kappa, b$ and $c$. Isotopic compositions are reported in $\delta$ or in $\varepsilon$ units as a function of the isotopic masses. Successively xenon (Xe, normalization at mass $130, \kappa=0.8, b=60$ and $c=-65$; data from Lewis et al. 1987), titanium (Ti, normalization at mass $48, \kappa=1.004, b=0.08$ and $c=0$ for $\varepsilon$ units and $\kappa=1.07$, $b=25$ and $c=-35$ for $\delta$ units; data from Hoppe et al. 1994, grain 071, and from Niederer et al. 1980, respectively), molybdenum (Mo, double normalization at masses 96 and 98, $\kappa=1.007, b=-0.8$ and $c=0.2$; data from Dauphas et al. 2002) and Samarium (Sm, double normalization at masses 148 and 150, $\kappa=0.8, b=-10$ and $c=-20$; data from McCulloch \& Wasserburg 1978b).

as for example its stabilization by a third body. The stabilization of the energetic molecule is certainly not the only way to retrieve the reactants from a chemical system and thus to give rise to a non-mass-dependent isotopic effect. As long as there is a competition between the isotopic exchange and the removal rates i.e. as long as two channels are open (see Appendix), the products of the reaction should be isotopically fractionated in a non-mass-dependent manner.
In a medium where high density reactive species or ions are present, the formation of energetic molecules able to react or to condense on a solid phase is markedly enhanced. Since these energetic molecules rarely appear spontaneously in a thermal gas, photochemistry or irradiation by hot atoms should promote this type of isotopic fractionation. 


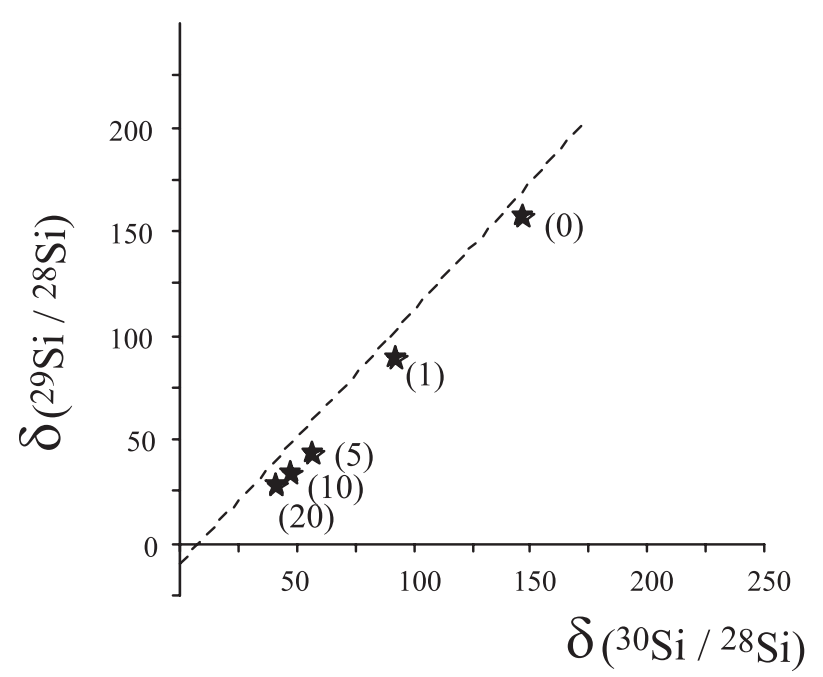

Fig. 2. The silicon isotopic variations (expressed in $\delta$ units) measured in the $\mathrm{SiC}$ grains extracted from meteorites are compared with calculated variations expected at different pressures for $\kappa=1.4, a=1$ and $b=4$. The slope 1.3 correlation observed in meteorites is reported as a dashed line and the calculations as stars with five different $P / P_{0}$ values indicated in parentheses $\left(P / P_{0}=0,1,5,10\right.$ and 20; see text at Eq. (30)). Data from Hoppe et al. (1994).

As far as the oxygen isotopic composition is concerned, the reactions between oxides and metastable oxygen species may yield this type of isotopic selection:

$\mathrm{Si}^{\mathrm{n}} \mathrm{O}+{ }^{\mathrm{m}} \mathrm{OH} \leftrightarrow \mathrm{Si}^{\mathrm{m}} \mathrm{O}+{ }^{\mathrm{n}} \mathrm{OH} \rightarrow \mathrm{SiO}_{2}+\mathrm{H}$

where $\mathrm{OH}$ is produced by the photolysis of water ( $\mathrm{m}$ and $\mathrm{n}$ stand for the isotopic mass values). The condensation of $\mathrm{SiO}_{2}$ on a solid can retrieve the energetic molecule $\mathrm{SiOOH}^{*}$ or $\mathrm{Si}(\mathrm{OH})_{2}^{*}$ (with $\mathrm{H}_{2} \mathrm{O}$ in place of $\mathrm{OH}$ in the reaction (31)).

In other elements, reactions between several oxides of the same element may also satisfy the requirement of the 3-body reaction scheme. Titanium is taken as an example:

${ }^{\mathrm{n}} \mathrm{Ti}+{ }^{\mathrm{m}} \mathrm{TiO}_{2} \leftrightarrow{ }^{\mathrm{m}} \mathrm{Ti}+{ }^{\mathrm{n}} \mathrm{TiO}_{2} \rightarrow \mathrm{Ti}_{2} \mathrm{O}_{2}+\mathrm{O} \rightarrow \mathrm{Ti}_{2} \mathrm{O}_{3}$.

The case of the rare gases is more puzzling since they rarely form stable molecules. It must be supposed that a selection mechanism favors chemical associations where a noble gas is stable. According to quantum mechanical calculations (Pauzat 2002) this may be the case between $\mathrm{Ar}$ and $\mathrm{H}_{3}^{+}$which should be stabilized as $\mathrm{ArH}_{3}^{+}$and $\mathrm{Ar}_{2} \mathrm{H}_{3}^{+}$. We can thus incorporate these molecules in a non-mass-dependent scheme:

${ }^{\mathrm{n}} \mathrm{Ar}+{ }^{\mathrm{m}} \mathrm{ArH}_{3}^{+} \leftrightarrow{ }^{\mathrm{m}} \mathrm{Ar}+{ }^{\mathrm{n}} \mathrm{ArH}_{3}^{+} \rightarrow \mathrm{Ar}_{2} \mathrm{H}_{3}^{+}$.

Although the complete calculation of the stability of $\mathrm{H}_{3}^{+}$bearing molecules has been performed for $\mathrm{ArH}_{3}^{+}$and $\mathrm{Ar}_{2} \mathrm{H}_{3}^{+}$, it seems possible that the other rare gases should behave similarly to Ar (Ellinger pers. com.).

In the case of the interactions between a solid and a (rare) gas, another process should be mentioned. The factors that control the rate of the various adsorption and desorption

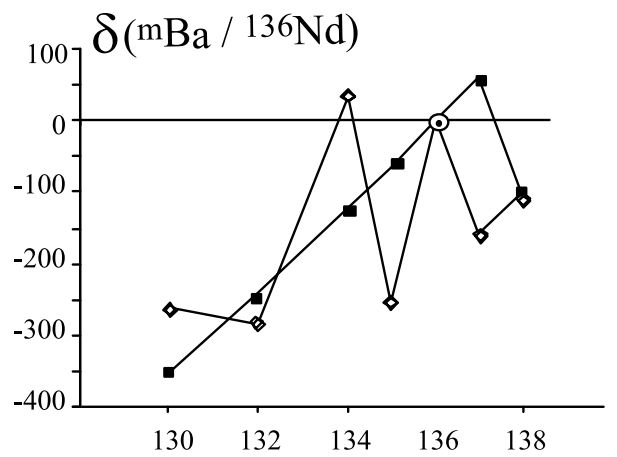

Ba Mass Number

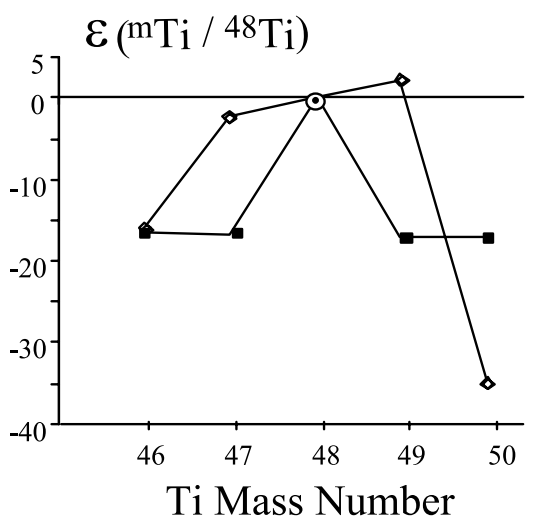

Fig. 3. Two examples of isotopic anomalies measured in meteorites which cannot be reconcile with the present theory (isotope ratios expressed in $\delta$ units, black dots for calculations and open symbols for data). Isotopic compositions are reported in $\delta$ or in $\varepsilon$ units as a function of the isotopic masses. Barium: Ba, normalization at mass 136 , $\kappa=2, b=0$ and $c=0$ (data from Ott \& Begemann 1990); titanium: Ti, normalization at mass $48, \kappa=0.995, b=0$ and $c=0$ (data from Niederer et al. 1980, 1984).

processes may have interesting similarities with the non-massdependent reaction scheme proposed here. The overall process for a precursor-mediated adsoption can be described as:

$I_{\mathrm{g}} \underset{k 1}{\stackrel{P}{\rightleftarrows}} I^{*} \underset{k 3}{\stackrel{k 2}{\longleftrightarrow}} I_{\mathrm{ad}}$

with $P$ the trapping probability into the weakly adsorbed state $\left(I^{*}\right)$ times the impingement rate and $k$ the various adsoption and desorption rates (subscripts " $g$ " for gas and "ad" for adsorbed). In many cases it has been shown that there is a finite probability of desorption from a precursor state $I^{*}$ rather than a direct passage in a stable adsorbed state. Thus the adsoption of energetic rare gases in a solid may also represent a potential direction for laboratory experiments.

\section{Nuclear or chemical effects?}

There are numerous examples demonstrating that nuclear effects are indeed present in meteoritic minerals and it is not the aim of this paper to claim that all isotopic anomalies result from the non-mass-dependent isotopic fractionation effect 


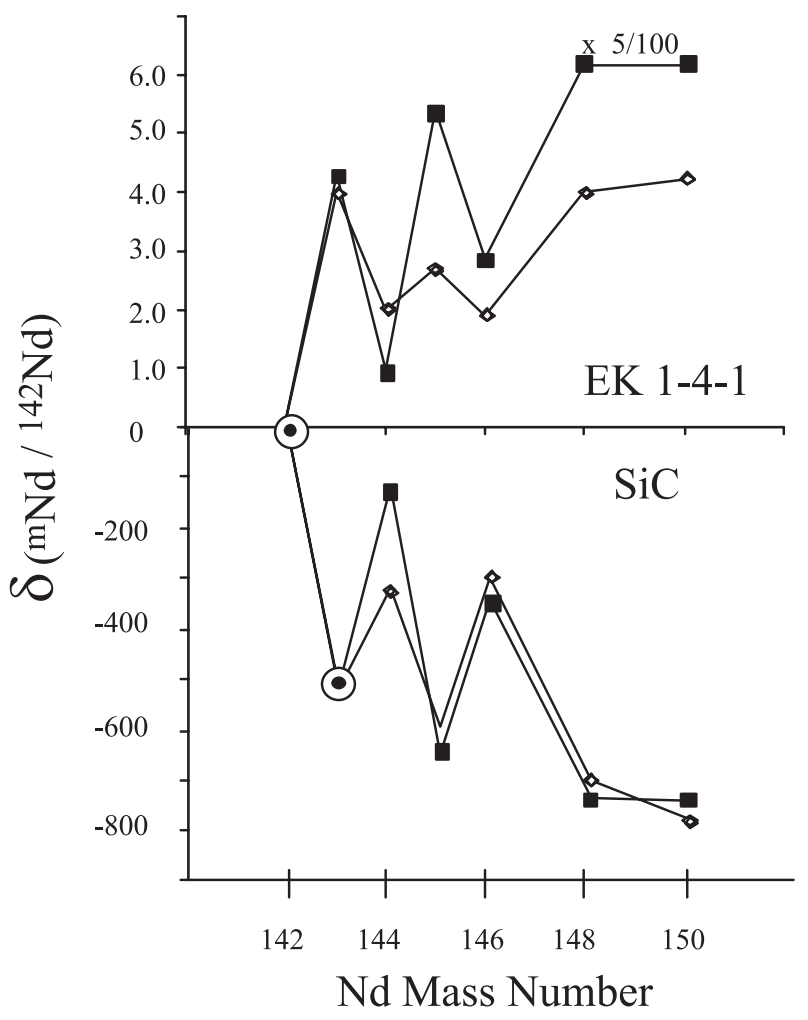

Fig. 4. Neodymium isotopic anomalies in the refractory inclusion EK 1-4-1 and in $\mathrm{SiC}$ (Nd, normalization at mass 142; data from McCulloch \& Wasserburg 1978a and from Richter et al. 1992). The calculation is performed by adjusting the parameter $\kappa$ in order to reproduce the negative $\delta^{143} \mathrm{Nd}$ (as shown by the two symbols at mass 142 and 143). All other masses are calculated with no additional adjustment of the parameters (black dots for calculations and open symbols for data). The positive pattern is arbitrarily divided by 20 (see text Sect. 5).

reported here. However, as often noted in the literature, interpreting all anomalies as the result of nuclear effects during supernova explosions is a difficult task. In many cases such as $\mathrm{C}$, $\mathrm{N}, \mathrm{Si}$, etc., and in the framework of the nuclear interpretation, the distribution of the isotopic compositions observed in meteoritic minerals must result from the contribution of grains condensed in the envelopes of different stars at different periods of the Galactic chemical evolution. For example, in the case of silicon isotopes, it seems almost impossible that the same star can produce the whole range of isotopic compositions defining the 1.3 slope correlation reported in Fig. 2. As illustrated by the calculation reported in Fig. 2, such an issue is overcome by the present treatment.

In many examples, positive and negative isotopic anomalies are observed for the same element but in different minerals. If these anomalies are nuclear, these observations have profound consequences for the origin of the chemical composition of our own solar system: isotopic anomalies cannot be viewed as a simple addition of freshly nucleosynthesized isotopes to the average, isotopically normal, solar system matter because such an addition would yield systematic isotopic enhancements. On the contrary, it must be supposed that the average solar system composition results from a thorough mixture of isotopic anomalies.

The present theory offers an alternative interpretation to this difficulty because the 3-body reaction scheme can, in principle yields positive or negative anomalies.

The mass balance calculation between the two channels can be performed as follows:

$2 x=x^{\prime}+x^{\prime \prime}$

with $x, x^{\prime}$ and $x^{\prime \prime}$ the abundance of a given isotope in the reactants (in I and IM), in the stabilization of the energetic molecule and in the products of the dissociation of the energetic molecule, respectively:

$$
\begin{array}{ll}
\mathrm{I}+\mathrm{IM} \rightarrow \mathrm{IIM}^{*} & (x) \\
\mathrm{IIM}^{*}+\mathrm{N} \rightarrow \mathrm{IIM}+\mathrm{N} & \left(x^{\prime}\right) \\
\mathrm{IIM}^{*} \rightarrow \mathrm{I}+\mathrm{IM} & \left(x^{\prime \prime}\right) .
\end{array}
$$

The treatments to calculate $x^{\prime}$ from $x$ were those given previously by (11), (27) and (28) (see also the two tables). Thus, when the isotopic pattern for the stabilized IIM molecule is calculated, the corresponding pattern for I+IM in (37) can be calculated with no additional assumption. The $\mathrm{Nd}$ isotopic anomalies are taken as an example of such a mass balance calculation.

For this element a slight positive isotopic anomaly was observed in the refractory inclusion EK 1-4-1 of the Allende meteorite and a large negative anomaly in $\mathrm{SiC}$. Let us assign the negative anomaly to the dissociation of the energetic molecule and the positive one to its stabilization. To reinforce the value of this calculation, the numerical results reported in Fig. 4 depend on only one parameter $\left(\kappa\right.$. The negative $\delta^{m} \mathrm{Nd}$ at mass $m=143$ was chosen to adjust $\kappa(\kappa=2.96)$. All the mass-dependent parameters were ignored i.e. $a=b=c=0$. In other terms, the only isotope adjusted in the calculation is at mass 143 while all the others (at mass 144, 145, 146,148 and 150 for the negative $\delta$ pattern and at mass 143,144, 145, 146, 148 and 150 for the positive $\delta$ pattern) are calculated rigorously. As reported in Fig. 4, the calculation shows close similarity to observations.

The calculated positive pattern is nevertheless enhanced in $\delta^{m} \mathrm{Nd}$ by a factor of 20 relative to the observed pattern; for example the calculated $\delta^{143} \mathrm{Nd}$ is $+80 \%$ as compared to the observed value of $+4 \%$. This can be interpreted as a dilution with isotopically normal $\mathrm{Nd}$ since - as already mentioned - such a dilution process does not change the relative position of the isotopic compositions in the anomalous pattern.

It is remarkable that this calculation reproduces not only the two patterns at all masses, but also that the large negative isotopic anomalies (in the range of $-500 \%$ ) correspond to moderate positive one (in the range of $+50 \%$ ). This does not imply that the two types of grains carrying the anomalies are co-genetic but that they can result from a similar isotopic fractionation process.

Compared with the nuclear interpretation, the present approach faces two types of difficulties. First, one can calculate numerous isotopic anomalies with the present treatment that are not observed in meteorites. Second, the chemical reactions at the origin of the calculated effects are not identified. The two 
difficulties are linked since the parameters of the isotopic fractionation correspond probably to a given chemical reaction (as shown for ozone). The situation is probably even more complicated because nuclear effects and non-mass-dependent effects could be intimately associated in the meteoritic minerals.

The concept of presolar grains (i.e. grains carrying isotopic anomalies) is not questioned by the present theory since it is not possible to decide if the chemical non-mass-dependent process took place (or not) during the formation of our own solar system, especially during the T-Tauri phase of the early Sun.

\section{Conclusions}

It is presently proposed that in chemical reactions, when isotopes are indistinguishable, the rate constant $k_{\mathrm{D}}$ for the dissociation of an energetic molecule should be written as:

$k_{\mathrm{D}, \mathrm{X}-\mathrm{XM}}=1 / 2\left[k_{1, \mathrm{X}-\mathrm{XM}}+k_{2, \mathrm{X}-\mathrm{XM}}\right]$

contrary to the classical isotopic fractionation theory where this rate constant is written as:

$k_{\mathrm{D}, \mathrm{X}-\mathrm{XM}}=1 / 2\left[k_{1, \mathrm{X}-\mathrm{XM}}+k_{1, \mathrm{X}-\mathrm{XM}}\right]=k_{1, \mathrm{X}-\mathrm{XM}}$.

Using this unique assumption, simple numerical applications using the classical approximations of the isotopic fractionation theory reproduce several isotopic anomalies found in meteoritic minerals. A physical interpretation of Eq. (38) has been proposed by Robert \& Camy-Peyret (2001). However, this interpretation seems not to be able to account for the measured ozone isotopic compositions (R. Marcus personal communication). It is thus possible that the relation (38) reflects the numerical consequences of an unrecognized quantum mechanical effect, linked to a property of the interaction between indistinguishable isotopes. If this assumption is correct, it should be possible to reproduce some of these isotopic anomalies in the laboratory, likely during the condensation (and cooling) of high temperature solids from a gas.

\section{Appendix}

The isotopic effect proposed by Robert \& Camy-Peyret (2001) can be illustrated by comparing - in a 3-body reaction scheme reactions involving distinguishable and indistinguishable isotopes. Two situations can be envisaged:

$\underline{\text { Situation } 1}$

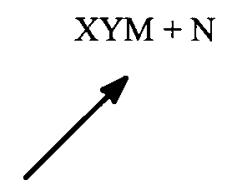

\section{$\mathrm{X}+\mathrm{YM} \rightarrow \mathrm{XYM}^{*}$}

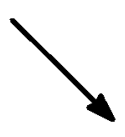

$\mathrm{X}+\mathrm{YM}($ or $\mathrm{Y}+\mathrm{XM})$

$\mathrm{P}(\mathrm{B})$
Situation 2

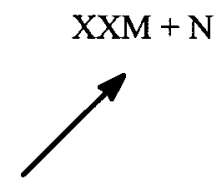

$\mathrm{P}(\mathrm{A})$

\section{$\mathrm{X}+\mathrm{XM} \rightarrow \mathrm{XXM}^{*}$}

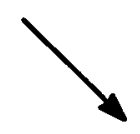

$$
\mathrm{X}+\mathrm{XM}
$$

with $\mathrm{X}$ and $\mathrm{Y}$ two isotopes of the same element, $\left({ }^{*}\right)$ standing for the energetic molecule, $\mathrm{N}$ the third body and $\mathrm{P}$ the probabilities of the two channels. When isotopes are distinguishable (Situation 1)

$$
\begin{aligned}
& \mathrm{P}(\mathrm{A})=P 1 \\
& \mathrm{P}(\mathrm{B})=1-P 1 .
\end{aligned}
$$

When indistinguishable (Situation 2):

$\mathrm{P}(\mathrm{A})=1 / 2(P 1+P 2)$

$\mathrm{P}(\mathrm{B}): 1 / 2[(1-P 1)+(1-P 2)]$.

The masses of the different isotopomers are ignored here for simplicity. When isotopes are distinguishable, there is only one single value of the impact parameter yielding $\mathrm{YXM}^{*}$ which can be stabilized, hence one probability $P 1$. When isotopes are indistinguishable, there are two values for the impact parameter (hence two probabilities $P 1$ and $P 2$ ) yielding two indistinguishable energetic molecules $\mathrm{XXM}^{*}$ which can be stabilized. For a situation where an energetic molecule is not formed during the collision between $\mathrm{X}$ and $\mathrm{XM}$, the two values for the impact parameter correspond to the scattering of $\mathrm{X}$ at the two angles $\theta$ and $\pi-\theta$ expressed in the laboratory coordinates (as detailed in Robert \& Camy-Peyret 2001).

Since in quantum mechanics - and contrary to classical mechanics - the two values of the impact parameter may have different probabilities, one has: $1 / 2(P 1+P 2) \neq P 1$.

Such an inequality is at the origin of the non-massdependent isotopic effect.

However, as noted by R. Marcus (pers. com.) this interpretation implies that the lifetime of the energetic molecule $\mathrm{XXM}^{*}$ is related to the value of the impact parameter from which the molecule has been formed. In other terms, the energetic molecule should not "forget" the conditions of its formation. This issue remains uncertain since, after its formation by the collision X-XM, the energetic molecule rotates and redistributes its internal energy in a chaotic manner. No quantum calculations of such an effect have yet been performed.

Acknowledgements. I would like to thank Samuel Epstein who passed away just two years ago; he let me work on the idea developed in this 
paper during my post-doctoral fellowship at Caltech in 1984 (I was supposed to work on cherts!) and ever since he encouraged me to publish it. Now it's done. Dr. R. Marcus reviewed this paper and I would also like to thank him for making me understand the conditions and limits of my interpretation. Our discussions, along with Dr. Micallef, have greatly improved this text.

\section{References}

Dauphas, N., Marty, B., \& Reisberg, L. 2002, ApJ, 565, 640

Clayton, R. N., Grossman, L., \& Mayeda, T. K. 1973, Science, 182, 485

Clayton, D. D., Deneault, E. A.-N., \& Meyer, B. S. 2001, ApJ, 562, 480

Clayton, D. D., Arnett, D., Kane, J., \& Meyer, B. S. 1997, ApJ, 486, 824

Gao, Q. Y., \& Marcus, R. A. 2001, Science, 293, 5528, 259

Gao, Q. Y., \& Marcus, R. A. 2001, J. Chem. Phys., 116, 137

Hathorn, B. C., \& Marcus, R. A. 1999, J. Chem. Phys., 111, 4087

Hathorn, B. C., \& Marcus, R. A. 2000, J. Chem. Phys., 113, 9497

Hoppe, P., Amari, S., Zinner, E., Ireland, T., \& Lewis, R. S. 1994, ApJ, 430,870

Janssen, C., Guenther, J., Krankowsky, D., \& Mauersberger, K. 1999, J. Chem. Phys., III, 7179

Janssen, C., Guenther, J., Mauersberger, K., \& Krankowsky, D. 2001, Phys. Chem. Chem. Phys., 3, 4718

Janssen, C., Guenther, J., Krankowsky, D., \& Mauersberger, K. 2003, Chem. Phys. Lett., 367, 34

Lewis, R. S., Tang, M., Wacker, J. F., Anders, E., \& Steel, E. 1987, Nature, 326, 160

Nicolussi, G. K., Pellin, M. J., Lewis, R. S., et al. 1998, ApJ, 504, 492
Niederer, F. R., Papanastassiou, D. A., \& Wasserburg, G. J. 1985, Geochim. Cosmochim. Acta, 49, 835

Niederer, F. R., \& Papanastassiou, D. A. 1984, Geochim. Cosmochim. Acta, 481279

Niederer, F. R., Papanastassiou, D. A., \& Wasserburg, G. J. 1980, ApJ, 240, L73

Ott, U., \& Begemann, F. 1990, ApJ, 353, L57

Ott, U. 1993, Nature, 364, 25

McCulloch, M. T., \& Wasserburg, G. J. 1978a, ApJ, 220, L15

McCulloch, M. T., \& Wasserburg, G. J. 1978b, Geophys. Res. Lett., 5,599

Mauersberger, K. 1981, Geophys. Res. Lett., 8, 935

Mauersberger, K., Erbacher, B., Krankowsky, D., Günther, J., \& Nickel, R. 1999, Science, 283, 370

Mauersberger, K., Jrankowsky, D., \& Janssen, C. 2003, Space Sci. Rev., 106, 265

Pauzat, F. 2002, Pers. com. and oral presentation at Almaden Ct. San Jose USA

Richter, S., Ott, U., \& Begemann, F. 1992, Lunar Planet Sci., XXIII, 1147

Robert, F., Halbout, J., \& Baudon, J. 1988, Earth. Planet. Sci. Lett., 91, 231

Robert, F., \& Baudon, J. 1990, Earth. Planet. Sci. Lett., 98, 402

Robert, F., \& Camy-Peyret, C. 2001, Ann. Geophys., 19, 229

Thiemens, M. 1999, Science, 283, 341

Thiemens, M. H., \& Heidenreich III, J. E. 1983, Science, 219, 1013

Wasserburg, G. J., Papanastassiou, D. A., \& Lee, T. 1980, in Early Solar System Processes and the Present day Solar System, ed. D. Lal (Amsterdam: North-Holland), 144

Zinner, E. 1995, Nuclei in the Cosmos III (New-York: AIP Woodbury), 567 